\title{
Maternal obesity during pregnancy and premature mortality from cardiovascular event in adult offspring: follow-up of 1323275 person years
}

Rebecca M Reynolds professor of metabolic medicine ${ }^{1}$, Keith M Allan research fellow ${ }^{2}$, Edwin A Raja statistician ${ }^{2}$, Sohinee Bhattacharya lecturer in obstetric epidemiology ${ }^{2}$, Geraldine McNeill professor of public health nutrition ${ }^{2}$, Philip C Hannaford professor of primary care ${ }^{2}$, Nadeem Sarwar research fellow ${ }^{2}$, Amanda J Lee professor of statistics ${ }^{2}$, Siladitya Bhattacharya professor of obstetrics $^{2}$, Jane $\mathrm{E}$ Norman professor of maternal and fetal health ${ }^{3}$

${ }^{1}$ Endocrinology Unit, BHF/University Centre for Cardiovascular Science, University of Edinburgh, Queen's Medical Research Institute, Edinburgh EH14 6TJ, UK; ${ }^{2}$ Institute of Applied Health Sciences, University of Aberdeen, Polwarth Building, Foresterhill, Aberdeen AB25 2ZD, UK ; ${ }^{3} \mathrm{Tommy}$ 's Centre for Fetal and Maternal Health, Medical Research Council Centre for Reproductive Health, University of Edinburgh, Queen's Medical Research Institute, Edinburgh EH14 6TJ, UK

\begin{abstract}
Objectives To determine whether maternal obesity during pregnancy is associated with increased mortality from cardiovascular events in adult offspring.

Design Record linkage cohort analysis.

Setting Birth records from the Aberdeen Maternity and Neonatal databank linked to the General Register of Deaths, Scotland, and the Scottish Morbidity Record systems.

Population 37709 people with birth records from 1950 to present day.
\end{abstract}

Correspondence to: R Reynolds R.Reynolds@ed.ac.uk

Extra material supplied by the author (see http://www.bmj.com/content/347/bmj.f4539?tab=related\#webextra)

Appendix Supplementary table A: Hazard ratios and $95 \%$ confidence intervals for cardiovascular events in offspring with data available on adult socioeconomic status according to maternal BMI category

Video on bmj.com (see also http://bmj.com/video)

(3)

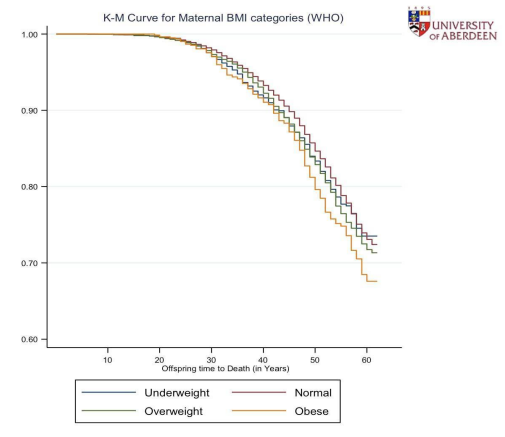

Main outcome measures Death and hospital admissions for cardiovascular events up to 1 January 2012 in offspring aged 34-61. Maternal body mass index (BMI) was calculated from height and weight measured at the first antenatal visit. The effect of maternal obesity on outcomes in offspring was tested with time to event analysis with Cox proportional hazard regression to compare outcomes in offspring of mothers in underweight, overweight, or obese categories of BMI compared with offspring of women with normal BMI.

Results All cause mortality was increased in offspring of obese mothers (BMI >30) compared with mothers with normal BMI after adjustment for maternal age at delivery, socioeconomic status, sex of offspring, current 
age, birth weight, gestation at delivery, and gestation at measurement of BMI (hazard ratio $1.35,95 \%$ confidence interval 1.17 to 1.55 ). In adjusted models, offspring of obese mothers also had an increased risk of hospital admission for a cardiovascular event (1.29, 1.06 to 1.57$)$ compared with offspring of mothers with normal BMI. The offspring of overweight mothers also had a higher risk of adverse outcomes.

Conclusions Maternal obesity is associated with an increased risk of premature death in adult offspring. As one in five women in the United Kingdom is obese at antenatal booking, strategies to optimise weight before pregnancy are urgently required.

\section{Introduction}

The obesity epidemic, with its attendant risk of cardiovascular and other chronic disease, has become a global threat to population health and a major public health burden. The prevalence of maternal obesity has risen rapidly in the past two decades. In the United States, about $64 \%$ of women of reproductive age are overweight and $35 \%$ are obese, ${ }^{1}$ with a similar pattern in Europe. ${ }^{2}$ The link between an adverse intrauterine environment and disease in later life is well documented, with many studies showing associations between low birth weight (a surrogate measure of intrauterine growth) and subsequent death from cardiovascular disease. ${ }^{3}$ Evidence from animal models and emerging data from humans suggest that maternal obesity also creates an adverse in utero environment, with long term "programmed" detrimental effects for the offspring. ${ }^{45}$ Several studies have shown associations between maternal obesity and obesity in the offspring, whether it is assessed in the neonatal period ${ }^{6}$ or in adulthood,

independent of current lifestyle and shared familial (genetic and environmental) factors. ${ }^{8}$ Recent studies have reported associations between higher maternal weight before pregnancy and biomarkers of future atheromatous disease in the offspring, including higher blood pressure and dyslipidaemia, in childhood ${ }^{9}$ and in young adulthood. ${ }^{10}$ Whether this translates into a long term increased risk of cardiovascular disease and death for the offspring is unknown. This is largely because of a lack of suitable cohorts with available data on maternal weight during pregnancy and with long enough follow-up of the offspring. To our knowledge there has been only one small published study reporting increased death from coronary heart disease in 3302 Finnish men who were thin at birth and whose mothers had a high body mass index (BMI) during pregnancy, with the finding restricted to mothers who were of short stature. ${ }^{11}$

We hypothesised that maternal obesity is associated with increased risk of death from cardiovascular events in their adult offspring. To test this we examined hospital admissions for cardiovascular events (including both coronary events and other vascular events) and death rates in 37709 individuals aged 34-61 whose mothers' BMI was recorded in pregnancy. We used a large database of pregnancy data, the Aberdeen Maternity and Neonatal Databank (AMND), linked to national death and morbidity records to generate 1323275 person years of follow-up.

\section{Methods}

Since 1950, the databank has collected information about pregnancy related events in women living in Aberdeen, Scotland; a relatively stable population. Completeness of the database is checked annually against the number of deliveries recorded in the National Health Service (NHS) records office. Validity of data entry is verified by numerous consistency checks. The background and details of the database are described in their website (www.amnd.ac.uk).
In this cohort analysis, the exposure was maternal obesity during pregnancy and the outcomes were all cause mortality and hospital admission with a cardiovascular event (defined below) in adult offspring. All women who delivered a live singleton baby at term (>37 weeks' gestation) between 1950 and 1976 and who had their weight recorded at their first antenatal visit were identified from the databank. The women were grouped according to their BMI, calculated from height and weight measurements taken at the first antenatal visit, by using World Health Organization criteria: underweight (BMI < 18.5), normal weight (BMI 18.5-24.9), overweight (BMI 25-29.9), and obese (BMI >30). After we examined the distribution, we deemed heights valid if they were within the range $1.22 \mathrm{~m}$ to $2.20 \mathrm{~m}$ and valid weights were in the range $28 \mathrm{~kg}$ to $179 \mathrm{~kg}$. Relevant details about the index pregnancy were retrieved from the databank, including maternal age, parity, husband/partner's social class, gestation at delivery, birth weight, sex of baby, and date of birth of baby, from which we calculated current age. Offspring's birth records were linked to the General Register of Deaths, Scotland, and the Scottish Morbidity record systems (SMR01) of the Information and Services Division (ISD), NHS Scotland, by probability matching on name, date of birth, and postcode (zip code) to identify cause specific death and all hospital admissions for cardiovascular events.

\section{Definition of cardiovascular disease and death}

We defined cardiovascular events in the offspring according to hospital discharge data codes (ICD (international classification of diseases) versions 9 and 10) consistent with angina (I20), myocardial infarction (I21-I23, I25), stroke (I61, I63, I64), other cerebrovascular disease (I60, I62, I65-69, G45 (transient ischaemic attack), peripheral artery disease (I70-I79), and other cardiovascular disease (I00, I01, I02, I05-I13, I15, I26-I28, I30-I52, I80- I89, I95, I97, I98, I99, Z95, R074, M31). We created a summary variable if an admission for any of these outcomes occurred. Years to event was calculated as the difference between the date of birth and either the date of death or first cardiovascular event up to 1 January 2012 when follow-up ceased.

\section{Statistical analysis}

Data were analysed with Stata (StataCorp, version 11, College Station, TX, USA). We tested for differences in maternal and offspring characteristics according to BMI category with analysis of variance for continuous variables and $\chi^{2}$ test for categorical variables. To evaluate the relation between maternal BMI and offspring mortality/cardiovascular events, we performed two sets of analyses in which we modelled BMI as both a categorical and a continuous variable.

For analyses of BMI as a categorical variable, we generated a Kaplan-Meier survival curve without accounting for clustering of offspring within a woman. Furthermore, taking into account clustering and other covariates, we tested the effect of maternal obesity and offspring outcome by time to event analysis, using Cox proportional hazard regression to estimate the hazard ratio (as relative risk) for death or cardiovascular disease in the offspring of mothers who were underweight, overweight, or obese in pregnancy according to their BMI compared with women with normal BMI. We estimated robust standard errors after adjusting for multiple offspring clustered within mothers. Analyses were also adjusted for potential confounding factors including maternal age at delivery, gestation when weight was measured, social class, parity, sex of offspring, gestation at delivery, birth weight, and current age of offspring. In the first analysis we included all women (and their children) with the 
first maternal weight measured at any time during pregnancy. We then repeated the analysis in the subset of women with first weight was recorded before 20 weeks' gestation to avoid the additional influences of gestational weight gain, which might be an independent risk factor ${ }^{910}$ for the outcomes of interest. We then investigated whether maternal obesity was associated with premature $($ age $<55)$ death in offspring. In a smaller supplementary subset we repeated the analyses adjusting for current socioeconomic status of offspring, which had been assessed in a subset of the offspring born in 1950-55 according to the registrar general's classification of occupations with a six point scale ranging from unskilled, semiskilled manual, skilled manual, skilled non-manual, managerial to professional (fig 1). $\Downarrow$

In the second set of analyses, to model the non-linear relation between maternal BMI as a continuous variable and mortality among offspring, we adopted the restricted cubic spline (RCS) procedure. ${ }^{12}$ This consisted of many polynomial line segments within the range of maternal BMI. The boundaries of the line segments are called knots. We considered seven knots for the model at the $2.5,18.33,34.17,50,65.83,81.67$ and 97.5th centiles. The corresponding maternal BMI values were 18.29 , $20.5,21.72,22.84,24.08,25.89$ and 31.37 , respectively. The mean maternal BMI value in the data was 22.86. Therefore we took the maternal BMI value of 23 as the reference value and compared offspring mortality with the value of other knots to assess the risk of death and construct the odds ratio graph.

\section{Results}

Table $1 \Downarrow$ shows the characteristics of the 28540 women and their 37709 offspring. Among the mothers, 21\% ( $n=5993)$ were overweight and $4 \%(\mathrm{n}=1141)$ were obese. Obese women were older, of greater parity, and lower social class. The offspring of obese women had a significantly higher birth weight but were younger (at the time of record linkage) than those in the other groups. The gestation at which weight was first measured in the pregnancy was significantly later in obese women, both in the whole cohort and the subset of women $(n=22136,59 \%)$ in whom weight was recorded before 20 weeks. The characteristics of women whose weight was measured in the first half of pregnancy were otherwise similar to the complete dataset.

\section{Maternal obesity and offspring death}

Among the 37709 offspring there were 6551 deaths from any cause. The leading cause of death was cardiovascular disease ( $24 \%$ of deaths in men and $13 \%$ in women) and cancer (26\% of deaths in men and $42 \%$ in women). Table $2 \Downarrow$ shows the hazard ratios and $95 \%$ confidence interval for death in offspring according to category of maternal BMI. There was a significant increase in all cause mortality (fig 2) in the offspring of mothers who were overweight or obese. $\Downarrow$ The same pattern was found in both the entire cohort and the restricted dataset with BMI measurements taken before 20 weeks. The hazard ratios increased after adjustment for gestation when weight was measured, maternal age at delivery, social class, current age of offspring, birth weight, and sex of offspring (hazard ratio 1.35, $95 \%$ confidence interval 1.17 to 1.55 ).

Table $2 \Downarrow$ also shows that maternal overweight and obesity was significantly associated with all cause premature mortality in offspring. There was no relation between birth weight and all cause mortality and no interaction between birth weight and maternal obesity on outcomes.

\section{Maternal obesity and cardiovascular events in offspring}

Overall, $7.6 \%$ of the offspring had been admitted to hospital with a cardiovascular event. In the complete dataset there was a significant association between maternal overweight or obesity and increased cardiovascular events in offspring, when we combined all hospital admissions for cardiovascular disease (table $3 \Downarrow$ ). There were also significant associations between maternal overweight and other cerebrovascular disease, peripheral artery disease, and other cardiovascular disease in the offspring and between maternal obesity and other cardiovascular disease. Similar patterns were seen in the restricted dataset of women with BMI recorded in early pregnancy (table 4 ) $\Downarrow$ and when the analyses included adjustment for current socioeconomic status (see supplementary table A in appendix).

\section{Exploring non-linear relation of maternal BMI with offspring death}

Using a restricted cubic spline procedure, we modelled the non-linear relation between offspring mortality rate and maternal BMI. Figure 3 shows that the odds of death in offspring was greater among mothers with low or high BMI compared with those with BMI of 23. $\Downarrow$ From figure 3 we can infer that the chances of death among the offspring were lowest among mothers with a BMI of 24-28.

\section{Discussion}

With the rising rates of excess weight among pregnant women, ${ }^{2}$ our findings of an association between maternal overweight and obesity and premature death in the adult offspring is a major public health concern. The offspring of mothers with increased BMI also experienced significantly more hospital admissions from all cardiovascular events combined. The observed relations were independent of several confounders, which could reflect the prenatal and postnatal environment, including maternal social class, maternal parity, and current age and sex of offspring. Our results suggest that the intrauterine environment has a crucial and long lasting effect on risk of premature mortality in offspring.

The developmental origins of health and disease hypothesis proposes that adverse conditions in utero during critical developmental periods lead to lifelong changes in body composition and physiology, with resultant adverse health in adulthood. ${ }^{3}$ The association between development in early life and death from coronary heart disease or cancer has been widely demonstrated in relation to low birth weight and/or maternal undernutrition. ${ }^{313}$ The influence of maternal obesity, which is now a more prevalent problem in developed countries and an emerging problem in countries undergoing economic transition, has not been studied in detail. We have shown that maternal obesity is a risk factor for all cause premature mortality in adult offspring and that this is independent of current socioeconomic status.

\section{Possible physiological mechanisms}

What mechanisms could link maternal obesity to death from cardiovascular disease in her offspring? Pregnant overweight women have increased circulating concentrations of inflammatory cytokines, non-esterified fatty acids, and amino acids and increased insulin resistance. ${ }^{14}$ Thus, there is a potential for increased nutrient supply to the developing fetus, with compensatory fetal hyperinsulinaemia and increased fetal 
adiposity. ${ }^{15}$ Animal data support the "developmental overnutrition hypothesis," which proposes that such conditions result in permanent changes in appetite control, neuroendocrine functioning, and/or energy metabolism in the offspring, leading to risk of adiposity, with accompanying cardiovascular risk factors, later in life. ${ }^{16}$ Intrauterine conditions might also cause lifelong alterations of key hormonal axes, such as the hypothalamic-pituitary-adrenal axis, predisposing to cardiovascular risk factors. ${ }^{17}$ Both animal and human studies have shown associations between maternal obesity and increased cardiovascular risk factors such as higher blood pressure, dyslipidaemia, and higher insulin concentrations in the offspring. ${ }^{4-10}$ The animal models have also shown structural changes in the heart and vasculature of the offspring in response to maternal overnutrition. These changes include endothelial dysfunction, ${ }^{18}$ increased sympathetic tone, ${ }^{19}$ and accumulation of connective tissue in the heart and myocardial fibrosis ${ }^{20}$; changes that would be expected to impair contractile function of the heart in offspring, predisposing them to later cardiac dysfunction. Furthermore, the potential role of epigenetic processes in the early life origins of disease is being increasingly recognised. Recent studies in primates have shown global and gene specific alterations in DNA methylation and histone modifications after maternal exposure to a high fat diet. ${ }^{21}$ Recent data in humans suggest that gene methylation changes in DNA extracted from umbilical cord tissue are associated with later childhood obesity. ${ }^{22}$ Such findings, if replicated, could allow early identification of high risk individuals.

\section{Strengths and weaknesses}

We cannot separate the direct effects of maternal obesity on the developing child from shared genetic and postnatal lifestyle influences in childhood and adulthood on obesity and cardiovascular risk. For example, there is evidence that obesity, unhealthy diets, and lack of exercise cluster in families. ${ }^{23} 24$ Regarding socioeconomic status, we were able to adjust for current socioeconomic status in a small subset of the adult offspring, though the limited sample size reduced study power and abolished significance. With offspring obesity, it is well recognised that there is a link between socioeconomic status and other adult lifestyle variables including obesity. While some studies have shown that the influence of maternal obesity on offspring obesity is independent of adult lifestyle factors, ${ }^{7}$ two studies showing associations between maternal obesity and cardiovascular risk factors had attenuated results after adjustment for current obesity levels. ${ }^{9}{ }^{10}$ As obesity in offspring is closely correlated to maternal obesity, however, further adjustment for offspring obesity might not be informative. Further work in animal models is necessary to disentangle the prenatal effects of maternal obesity from postnatal environmental effects. One limitation of our study is that no information was available on paternal obesity, and there is now some evidence of programming of obesity through the paternal line. ${ }^{25}$ Nevertheless, our results are important, indicating that the offspring of obese mothers are a high risk group who should be assessed for cardiovascular risk, and actively encouraged to maintain a healthy lifestyle. This could avoid perpetuation of an intergenerational cycle of obesity.

A major strength of our study was its large size, with high quality detailed antenatal records. Height and weight were measured at the first antenatal visit, so calculation of BMI did not rely on self report of these measurements. Self report can lead to underestimation of BMI in overweight and obese individuals. ${ }^{26}$ Although there was an association between increased BMI and the gestational week when this was recorded, we adjusted for the latter in our analyses. We also found similar results when we included only women with measurements taken during the first half of pregnancy. As there is usually minimal weight gain during the first 16 weeks, ${ }^{27}$ this subgroup analysis is likely to reflect the influence of BMI at the time of conception rather than the effects of overall weight gain during pregnancy. Nevertheless, patterns of weight gain, even in early pregnancy, are variable, and studies with measurements of weight at different stages of pregnancy are needed to determine whether there are differing effects of gestational weight change rather than obesity per se. A further strength of our study was that we had long enough follow-up of offspring for cardiovascular events to occur, although the population was still quite young and so our study might have underestimated the size of the effect. There were indications of an increase in hospital admissions for specific cardiovascular events, although these often failed to reach significance, possibly because of limited statistical power. Further follow-up in the cohort as it ages will be informative. Linkage rates were greater than $80 \%$. The use of collated data from the Information and Services Division enabled us to capture events among offspring who moved from Aberdeen to another part of Scotland but not those occurring outside the country. Provided that the loss of information was unconnected with either maternal weight and death or cardiovascular disease in the offspring, the effect of the loss will have been to reduce our ability to find associations rather than introduce systematic bias. Offspring who were healthy, without any hospital admission, would have remained unlinked to the database. Our results, therefore, could represent an "unhealthy worker" effect. As we looked only at hospital admissions, we will have examined only more severe cardiovascular disease.

\section{Conclusions}

Our data build on the literature showing associations between excess maternal weight and adverse health in the offspring. They show an association between maternal obesity and all cause mortality in the offspring, findings that are independent of current socioeconomic status. Only $4 \%$ of women in our cohort were obese, far smaller than current levels in the US and UK. Our findings highlight the urgent need for strategies to prevent obesity in women of childbearing age and the need to assess the offspring of obese mothers for their cardiovascular risk.

We thank Katie Wilde, Data Management Team, University of Aberdeen, and Lynsey Waugh, Information and Services Division of NHS Scotland, for their help with data extraction and linkage. We also thank Cairns Smith and Heather Clark for their work with the Aberdeen Children of the 50s cohort.

Contributors: RMR designed the study, analysed the data, and wrote the manuscript; KMA analysed the data and edited the manuscript; EAR carried out the statistical analysis and edited the manuscript; SoB designed the study, assisted with data analysis, and edited the manuscript; GMcN, PCH, and JEN designed the study and edited the manuscript; NS edited the manuscript; AJL assisted with data analysis and edited the manuscript; SB designed the study, analysed the data, and edited the manuscript. SB is guarantor.

Funding: This work was supported by grants from the chief scientist office, Scotland, and Chest, Heart and Stroke, Scotland. We also acknowledge support from Tommy's and the British Heart Foundation. The funders had no role in study design, data collection and analysis, decision to publish, or preparation of the manuscript.

Competing interests: All authors have completed the ICMJE uniform disclosure form at www.icmje.org/coi_disclosure.pdf (available on request from the corresponding author) and declare: NS has worked 


\section{What is already known on this topic}

Obesity in pregnancy is associated with cardiometabolic risk factors in the offspring

Whether maternal obesity is also associated with increased premature death in offspring from cardiovascular events is unknown because of lack of information about maternal BMI in pregnancy or insufficient length of follow-up in offspring

\section{What this study adds}

Maternal obesity is associated with an increase in the risk of premature death and hospital admissions for cardiovascular events in her adult offspring

Offspring of overweight women also have higher risk of adverse events in later life

As one in five women in the UK is currently obese at antenatal booking, strategies to optimise weight before pregnancy are urgently required

for Pfizer since 2011, JEN has received research grants from (non-commercial) funding agencies for pregnancy related conditions (full list available on request); funding from UK government agencies for providing reports on maternal deaths; honorariums for book chapters and books in obstetrics and gynaecology; and fees for consultancy to a small drug company (Preglem) with an interest in obstetric/gynaecological drugs. JEN is an unpaid consultant to Hologic (who manufacture fibronectin among others).

Ethical approval: The study was approved by the North of Scotland research ethics service (REC reference: 10/S11034/15) and the steering committee of the AMND and the privacy advisory committee of ISD, Scotland.

Data sharing: No additional data available.

1 Flegal KM, Carroll MD, Kit BK, Ogden CL. Prevalence of obesity and trends in the distribution of body mass index among US adults, 1999-2010. JAMA 2012;307:491-7.

2 Heslehurst N, Rankin J, Wilkinson JR, Summerbell CD. A nationally representative study of maternal obesity in England, UK: trends in incidence and demographic inequalities in 619323 births, 1989-2007. Int J Obes (Lond) 2010:34:420-8.

3 Barker DJP. Fetal origins of coronary heart disease. BMJ 1995;311:171-4.

4 Drake AJ, Reynolds RM. Impact of maternal obesity on offspring obesity and cardiometabolic disease risk. Reproduction 2010;140:387-98.

5 Li M, Sloboda DM, Vickers MH. Maternal obesity and developmental programming of metabolic disorders in offspring: evidence from animal models. Exp Diabetes Res 2011;2011:592408.

6 Catalano PM, Presley L, Minium J, Hauguel-de MS. Fetuses of obese mothers develop insulin resistance in utero. Diabetes Care 2009;32:1076-80.

7 Reynolds RM, Osmond C, Phillips DI, Godfrey KM. Maternal BMI, parity, and pregnancy weight gain: influences on offspring adiposity in young adulthood. J Clin Endocrinol Metab 2010;95:5365-9.

8 Lawlor DA, Lichtenstein P, Fraser A, Langstrom N. Does maternal weight gain in pregnancy have long-term effects on offspring adiposity? A sibling study in a prospective cohort of 146,894 men from 136,050 families. Am J Clin Nutr 2011;94:142-8.

9 Fraser A, Tilling K, Macdonald-Wallis C, Sattar N, Brion MJ, Benfield L, et al. Association of maternal weight gain in pregnancy with offspring obesity and metabolic and vascular traits in childhood. Circulation 2010;121:2557-64.

10 Hochner H, Friedlander Y, Calderon-Margalit R, Meiner V, Sagy Y, Avgil-Tsadok M, et al. Associations of maternal prepregnancy body mass index and gestational weight gain with adult offspring cardiometabolic risk factors. The Jerusalem Perinatal Family Follow-Up Study. Circulation 2012;125:1381-9.

11 Forsen T, Eriksson JG, Tuomilehto J, Teramo K, Osmond C, Barker DJ. Mother's weight in pregnancy and coronary heart disease in a cohort of Finnish men: follow up study. BMJ 1997;315:837-40

12 Dupout WD. Statistical modelling for biomedical researchers. 2nd ed. Cambridge University Press, 2008.

13 Kajantie E, Osmond C, Barker DJP, Forsen T, Phillips DI, Eriksson JG. Size at birth as a predictor of mortality in adulthood: a follow-up of 350000 person-years. Int J Epidemiol 2005;34:655-63.
14 Ramsay JE, Ferrell WR, Crawford L, Wallace AM, Greer IA, Sattar N. Maternal obesity is associated with dysregulation of metabolic, vascular, and inflammatory pathways. $J$ Clin Endocrinol Metab 2002;87:4231-7.

15 Catalano PM, Ehrenberg HM. The short- and long-term implications of maternal obesity on the mother and her offspring. BJOG 2006;113:1126-33.

16 Taylor PD, Poston L. Developmental programming of obesity in mammals. Exp Physiol 2007;92:287-98.

17 Reynolds RM, Walker BR, Phillips DIW, Sydall HE, Andrew R, Whorwood CB, et al. Altered control of cortisol secretion in adult men with low birthweight and cardiovascular risk factors. J Clin Endocrinol Metab 2001;86:245-50.

18 Samuelsson AM, Matthews PA, Argenton M, Christie MR, McConnell JM, Jansen EH, et al. Diet-induced obesity in female mice leads to offspring hyperphagia, adiposity, hypertension, and insulin resistance: a novel murine model of developmental programming. Hypertension 2008;51:383-92.

19 Samuelsson AM, Morris A, Igosheva N, Kirk SL, Pombo JM, Coen CW, et al. Evidence for sympathetic origins of hypertension in juvenile offspring of obese rats. Hypertension 2010;55:76-82.

20 Huang Y, Yan X, Zhao JX, Zhu MJ, McCormick RJ, Ford Sp, et al. Maternal obesity induces fibrosis in fetal myocardium of sheep. Am J Physiol Endocrinol Metab 2010;299:E968-75.

21 Aagaard-Tillery KM, Grove K, Bishop J, Ke X, Fu Q, McKnight R, et al. Developmental origins of disease and determinants of chromatin structure: maternal diet modifies the primate fetal epigenome. J Mol Endocrinol 2008:41:91-102.

22 Godfrey KM, Sheppard A, Gluckman PD, Lillycrop KA, Burdge GC, McLean C, et al. Epigenetic gene promoter methylation at birth is associated with child's later adiposity. Diabetes 2011;60:1528-34.

23 Laskarzewski P, Morrison JA, Khoury P, Kelly K, Glatfelter L, Larsen R, et al. Parent-child nutrient intake interrelationships in school children ages 6 to 19: the Princeton School District Study. Am J Clin Nutr 1980;33:2350-5.

24 Simonen RL, Perusse L, Rankinen T, Rice T, Rao DC, Bouchard C. Familial aggregation of physical activity levels in the Quebec Family Study. Med Sci Sports Exerc 2002;34:1137-42.

25 Cooper R, Hypponen E, Berry D, Power C. Associations between parental and offspring adiposity up to midlife: the contribution of adult lifestyle factors in the 1958 British Birth Cohort Study. Am J Clin Nutr 2010;92:946-53.

26 Stommel M, Schoenborn CA. Accuracy and usefulness of BMI measures based on self-reported weight and height: findings from the NHANES \& NHIS 2001-2006. BMC Public Health 2009;9:421.

27 Fattah C, Farah N, O'Toole F, Barry S, Stuart B, Turner MJ. Body mass index (BMI) in women booking for antenatal care: comparison between selfreported and digital measurements. Eur J Obstet Gynecol Repro Biol 2009;144:32-4.

Accepted: 11 July 2013

\section{Cite this as: BMJ 2013;347:f4539}

This is an Open Access article distributed in accordance with the Creative Commons Attribution Non Commercial (CC BY-NC 3.0) license, which permits others to distribute, remix, adapt, build upon this work non-commercially, and license their derivative works on different terms, provided the original work is properly cited and the use is non-commercial. See: http://creativecommons.org/licenses/by-nc/3.0/. 


\section{Tables}

Table 1 | Characteristics of mothers and their offspring by maternal body mass index (BMI). Figures are means (SD) unless otherwise specified

\begin{tabular}{|c|c|c|c|c|c|}
\hline & \multicolumn{4}{|c|}{ Maternal BMI (WHO) category } & \multirow[b]{2}{*}{$P$ value } \\
\hline & $\begin{array}{l}\text { Underweight (1195 } \\
\text { offspring) }\end{array}$ & Normal (27 051 offspring) & Overweight (7913 offspring) & Obese (1550 offspring) & \\
\hline Age at delivery (years) & $24.9(4.6)$ & $25.2(5.1)$ & $26.7(5.7)$ & $27.9(6.0)$ & $<0.001$ \\
\hline Maternal BMI $\left(\mathrm{kg} / \mathrm{m}^{2}\right)$ & $17.7(0.73)$ & $22.0(1.6)$ & $26.8(1.3)$ & $32.9(3.3)$ & $<0.001$ \\
\hline \multicolumn{6}{|c|}{ Gestation when BMI was measured (weeks): } \\
\hline In all women & $13.7(3.9)$ & $16.8(5.8)$ & $19.5(7.3)$ & $20.7(7.8)$ & $<0.001$ \\
\hline $\begin{array}{l}\text { In subgroup measured before } 20 \\
\text { weeks }\end{array}$ & $13.2(3.0)$ & $14.3(3.3)$ & $14.7(3.4)$ & $14.9(3.4)$ & $<0.001$ \\
\hline \multicolumn{6}{|l|}{ No (\%) by parity of mother: } \\
\hline Primiparous & $704(59)$ & $14485(53)$ & $3265(41)$ & $458(30)$ & $<0.001$ \\
\hline Multiparous & $491(41)$ & $12566(47)$ & $4648(59)$ & $892(70)$ & \\
\hline \multicolumn{6}{|l|}{ No (\%) of mothers by social class: } \\
\hline I-IIla non-manual & $312(30)$ & $6305(28)$ & $1510(24)$ & $127(13)$ & $<0.001$ \\
\hline Illb manual-V & $719(70)$ & $16534(72)$ & $4717(76)$ & $889(87)$ & \\
\hline Gestation at delivery (weeks) & $40.3(1.4)$ & $40.4(1.4)$ & $40.4(1.4)$ & $40.4(1.5)$ & 0.01 \\
\hline Birth weight (g) & $3132(446)$ & $3299(456)$ & $3436(469)$ & $3536(483)$ & $<0.001$ \\
\hline No (\%) of male:female offspring & $610(51): 585(49)$ & 13829 (51):13 221 (49) & 4168 (52):3745 (48) & $808(53): 742(47)$ & 0.372 \\
\hline $\begin{array}{l}\text { Age of offspring (years) at date of } \\
\text { record linkage }\end{array}$ & $51.5(7.6)$ & $50.7(7.9)$ & $49.8(8.2)$ & $49.2(8.0)$ & $<0.001$ \\
\hline
\end{tabular}


Table 2| Hazard ratios and $95 \%$ confidence intervals for death after cardiovascular event in adult offspring according to maternal BMI category*

Hazard ratio $(95 \% \mathrm{Cl})$

No $(\%)$ of deaths

Unadjusted

Adjusted $\dagger$

Death in offspring of all women $(n=37709)$

\begin{tabular}{|c|c|c|c|}
\hline Mother underweight & $230(3.5)$ & $1.07(0.92$ to 1.24$)$ & $0.98(0.84$ to 1.14 \\
\hline Mother normal weight & $4690(72.6)$ & 1.00 & 1.00 \\
\hline Mother overweight & $1337(20.4)$ & $1.02(0.95$ to 1.09$)$ & $1.11(1.03$ to 1.19$)$ \\
\hline Mother obese & $294(4.5)$ & $1.18(1.03$ to 1.35$)$ & 1.35 (1.17 to 1.55$)$ \\
\hline \multicolumn{4}{|c|}{ Death in offspring of women with BMI measured $<20$ weeks ( $n=27277$ ) } \\
\hline Mother underweight & $214(4.5)$ & $1.06(0.91$ to 1.23 & $0.99(0.85$ to 1.16 \\
\hline Mother normal weight & $3542(74.1)$ & 1.00 & 1.00 \\
\hline Mother overweight & $860(18.0)$ & 1.11 (1.02 to 1.20 & 1.17 (1.08 to 1.28 \\
\hline Mother obese & $165(3.5)$ & 1.29 (1.08 to 1.53 & $1.42(1.19$ to 1.69 \\
\hline \multicolumn{4}{|c|}{ Offspring died before age 55} \\
\hline Mother underweight & $199(4.6)$ & $1.10(0.94$ to 1.29$)$ & $1.03(0.88$ to 1.21$)$ \\
\hline Mother normal weight & $3218(73.6)$ & 1.00 & 1.00 \\
\hline Mother overweight & $805(18.4)$ & $1.14(1.04$ to 1.24$)$ & $1.19(1.09$ to 1.30$)$ \\
\hline Mother obese & $152(3.5)$ & $1.29(1.08$ to 1.55$)$ & $1.40(1.17$ to 1.68$)$ \\
\hline \multicolumn{4}{|c|}{ Offspring died after age 55} \\
\hline Mother underweight & $15(3.7)$ & $0.71(0.40$ to 1.26$)$ & $0.63(0.35$ to 1.13$)$ \\
\hline Mother normal weight & $324(79.6)$ & 1.00 & 1.00 \\
\hline Mother overweight & $55(13.5)$ & $0.84(0.62$ to 1.12$)$ & $1.01(0.75$ to 1.37$)$ \\
\hline Mother obese & $13(3.2)$ & $1.27(0.70$ to 2.30$)$ & 1.77 (0.96 to 3.27$)$ \\
\hline
\end{tabular}

${ }^{*}$ Results from Cox proportional hazards model after adjustment of SE for multiple offspring within mothers.

†Adjusted for husband/partner's social class, gestational age, sex of baby and birth weight, when in gestation weight was measured, and mother's age at delivery. 
Table 3| Hazard ratios and $95 \%$ confidence intervals for hospital admissions for cardiovascular events in all adult offspring according to maternal BMI category*

\begin{tabular}{|c|c|c|c|}
\hline & \multirow[b]{2}{*}{ No $(\%)$ of events } & \multicolumn{2}{|c|}{ Hazard ratio $(95 \% \mathrm{Cl})$} \\
\hline & & Unadjusted & Adjusted $†$ \\
\hline \multicolumn{4}{|c|}{ All cardiovascular events combined } \\
\hline Mother underweight & $77(2.9)$ & 0.85 (0.68 to 1.07 ) & $0.80(0.63$ to 1.00$)$ \\
\hline Mother normal weight & $1921(71.7)$ & 1 & 1 \\
\hline Mother overweight & $566(21.1)$ & 1.07 (0.97 to 1.17$)$ & $1.15(1.04$ to 1.26$)$ \\
\hline Mother obese & $114(4.3)$ & 1.15 (0.95 to 1.39$)$ & 1.29 (1.06 to 1.57$)$ \\
\hline \multicolumn{4}{|l|}{ Angina } \\
\hline Mother underweight & $2(0.9)$ & 0.25 (0.06 to 1.02$)$ & $0.24(0.06$ to 0.99$)$ \\
\hline Mother normal weight & $166(73.8)$ & 1 & 1 \\
\hline Mother overweight & $45(20.0)$ & 0.99 (0.71 to 1.39$)$ & $1.05(0.74$ to 1.47$)$ \\
\hline Mother obese & $12(5.3)$ & 1.42 (0.79 to 2.55$)$ & $1.53(0.84$ to 2.81$)$ \\
\hline \multicolumn{4}{|l|}{ Myocardial infarction } \\
\hline Mother underweight & $6(2.0)$ & $0.56(0.25$ to 1.26$)$ & $0.53(0.23$ to 1.20$)$ \\
\hline Mother normal weight & $225(73.5)$ & 1 & 1 \\
\hline Mother overweight & $66(21.6)$ & $1.08(0.81$ to 1.42$)$ & $1.19(0.89$ to 1.58$)$ \\
\hline Mother obese & $9(2.9)$ & 0.78 (0.40 to 1.52$)$ & 0.95 (0.49 to 1.83$)$ \\
\hline \multicolumn{4}{|l|}{ Stroke } \\
\hline Mother underweight & $4(3.1)$ & 0.94 (0.35 to 2.57 ) & 0.84 (0.31 to 2.30$)$ \\
\hline Mother normal weight & $89(68.5)$ & 1 & 1 \\
\hline Mother overweight & $32(24.6)$ & $1.32(0.88$ to 1.98$)$ & $1.46(0.96$ to 2.20$)$ \\
\hline Mother obese & $5(3.8)$ & 1.10 (0.45 to 2.71$)$ & $1.04(0.37$ to 2.94$)$ \\
\hline \multicolumn{4}{|c|}{ Other cerebrovascular disease } \\
\hline Mother underweight & $3(2.0)$ & 0.64 (0.20 to 2.01$)$ & $0.64(0.20$ to 2.05$)$ \\
\hline Mother normal weight & $100(66.2)$ & 1 & 1 \\
\hline Mother overweight & $41(27.2)$ & 1.50 (1.04 to 2.15$)$ & $1.61(1.10$ to 2.36$)$ \\
\hline Mother obese & $7(4.6)$ & 1.36 (0.63 to 2.91$)$ & 1.54 (0.69 to 3.42$)$ \\
\hline \multicolumn{4}{|c|}{ Peripheral arterial disease } \\
\hline Mother underweight & $6(2.9)$ & 0.91 (0.40 to 2.06 ) & 0.90 (0.40 to 2.05$)$ \\
\hline Mother normal weight & $140(66.7)$ & 1 & 1 \\
\hline Mother overweight & $55(26.2)$ & $1.43(1.05$ to 1.95$)$ & $1.52(1.10$ to 2.10$)$ \\
\hline Mother obese & $9(4.3)$ & 1.24 (0.64 to 2.43 ) & 1.27 (0.61 to 2.62$)$ \\
\hline \multicolumn{4}{|c|}{ Other cardiovascular disease } \\
\hline Mother underweight & $63(2.9)$ & 0.85 (0.66 to 1.09 ) & 0.79 (0.61 to 1.02$)$ \\
\hline Mother normal weight & $1576(72.0)$ & 1 & 1 \\
\hline Mother overweight & $456(20.8)$ & 1.05 (0.94 to 1.16$)$ & $1.13(1.02$ to 1.26$)$ \\
\hline Mother obese & $94(4.3)$ & 1.15 (0.93 to 1.42$)$ & 1.31 (1.05 to 1.63$)$ \\
\hline
\end{tabular}

${ }^{*}$ Results from Cox proportional hazards model after adjustment for SE for multiple offspring within mothers.

†Adjusted for husbands' social class, gestational age, sex of the baby and birth weight, when in gestation weight was measured, and mother's age at delivery. 
Table 4| Hazard ratios and 95\% confidence intervals for cardiovascular events in adult offspring of mothers with BMI measured in early pregnancy, according to maternal BMI category*

\begin{tabular}{|c|c|c|c|}
\hline & No (\%) of events & \multicolumn{2}{|c|}{ Hazard ratio $(95 \% \mathrm{Cl})$} \\
\hline \multicolumn{4}{|c|}{ All cardiovascular events combined } \\
\hline Mother underweight & $70(3.7)$ & 0.83 (0.65 to 1.05$)$ & $0.79(0.62$ to 1.01$)$ \\
\hline Mother normal weight & $144(7.5)$ & 1 & 1 \\
\hline \multicolumn{4}{|l|}{ Angina } \\
\hline Mother underweight & $2(1.3)$ & $0.28(0.07$ to 1.11$)$ & 0.26 (0.06 to 1.07$)$ \\
\hline Mother normal weight & $121(79.6)$ & 1 & 1 \\
\hline Mother overweight & $21(13.8)$ & $0.81(0.51$ to 1.28$)$ & 0.84 (0.53 to 1.34$)$ \\
\hline \multicolumn{4}{|l|}{ Myocardial infarction } \\
\hline Mother underweight & $5(2.4)$ & $0.52(0.21$ to 1.27$)$ & $0.46(0.19$ to 1.14$)$ \\
\hline Mother normal weight & $162(78.3)$ & 1 & 1 \\
\hline Mother overweight & $36(17.4)$ & $1.04(0.72$ to 1.49$)$ & $1.19(0.82$ to 1.70$)$ \\
\hline Mother obese & $4(1.9)$ & $0.71(0.26$ to 1.93$)$ & $0.86(0.32$ to 2.32$)$ \\
\hline \multicolumn{4}{|l|}{ Stroke } \\
\hline Mother underweight & $4(4.4)$ & $1.02(0.37$ to 2.81$)$ & 0.89 (0.32 to 2.46$)$ \\
\hline Mother normal weight & $66(72.5)$ & 1 & 1 \\
\hline Mother overweight & $19(20.9)$ & 1.35 (0.81 to 2.25$)$ & $1.48(0.88$ to 2.48$)$ \\
\hline \multicolumn{4}{|c|}{ Peripheral arterial disease } \\
\hline Mother underweight & $6(4.2)$ & $1.02(0.45$ to 2.32$)$ & $0.96(0.42$ to 2.22$)$ \\
\hline Mother normal weight & $101(70.6)$ & 1 & 1 \\
\hline Mother overweight & $31(21.7)$ & $1.42(0.95$ to 2.12$)$ & 1.55 (1.02 to 2.34$)$ \\
\hline Mother obese & $5(3.5)$ & $1.41(0.57$ to 3.45$)$ & $1.61(0.66$ to 3.95$)$ \\
\hline \multicolumn{4}{|c|}{ Other cardiovascular disease } \\
\hline Mother underweight & $56(3.6)$ & $0.81(0.62$ to 1.06$)$ & $0.78(0.59$ to 1.01$)$ \\
\hline Mother normal weight & $1180(75.2)$ & 1 & 1 \\
\hline Mother overweight & $282(20.0)$ & $1.10(0.97$ to 1.25$)$ & $1.17(1.02$ to 1.33$)$ \\
\hline Mother obese & $51(3.3)$ & 1.22 (0.92 to 1.62$)$ & 1.33 (1.00 to 1.77$)$ \\
\hline
\end{tabular}

*Results from Cox proportional hazards model after adjustment for SE for multiple offspring within mothers.

†Adjusted for husbands' social class, gestational age, sex of the baby and birth weight, gestation weight measured, and mother's age at delivery. 


\section{Figures}

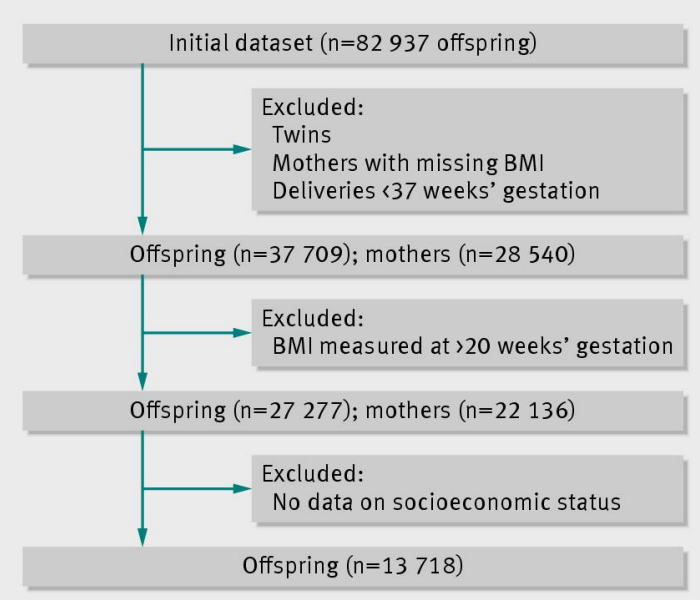

Fig 1 Identification of samples for analysis

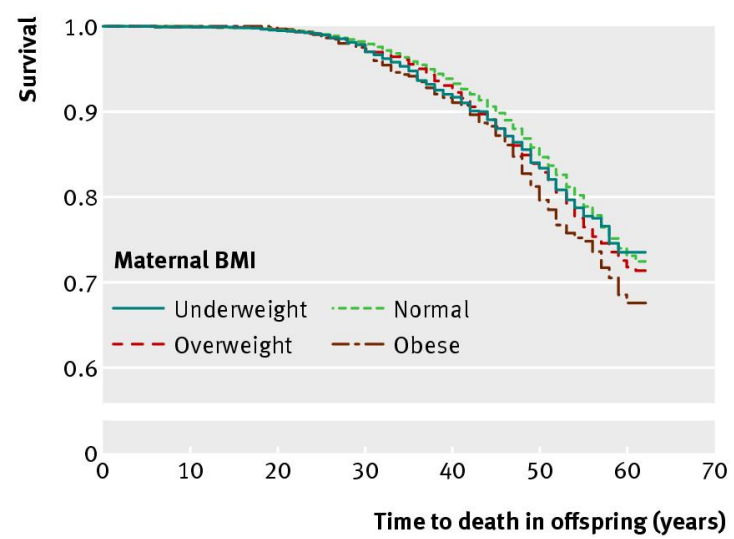

Fig 2 Kaplan-Meier curves for death rates in offspring according to maternal BMI category showing increased adjusted all cause premature mortality in offspring of obese mothers (BMI >30)

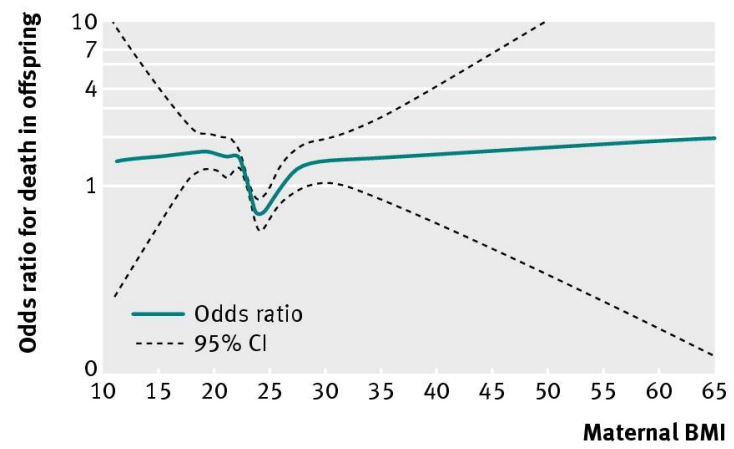

Fig 3 Non-linear relation of maternal BMI with mortality in offspring, showing that odds of offspring death was more among mothers with low or high BMI before pregnancy compared with mothers with BMI of 23 